\title{
Modifications of dental adhesive systems
}

\begin{abstract}
The ultimate goal of a bonded restoration is to attain an intimate adaptation of the restorative material with the dental substrate. This task is difficult to achieve as the bonding process for enamel is different from that for dentin. That is, dentin is much humid and more organic than enamel. This humid and organic nature of dentin makes bonding extremely difficult. The purpose of this work is to review the main concepts regarding various adhesive systems.

Keywords: adhesive, bonding agent, dentin, restorative dentistry, resin composite
\end{abstract}

Volume 9 Issue I - 2018

\author{
Tamer M Hamdy \\ Department of Endodontics, Restorative \& Dental Materials, \\ National Research Centre (NRC), Egypt
}
Correspondence: Tamer M Hamdy, Department of Endodontics, Restorative \& Dental Materials, National Research Centre (NRC), El Bohouth St., I 2622 Dokki, Giza, Egypt, Email dr_tamer_hamdy@yahoo.com

Received: September 27, 2017 | Published: January 02, 2018

\begin{abstract}
Abbreviations: CQ, camphorquinone; PPD, 1-phenyl-1,2 Propanedione; BPO, benzoyl peroxide; MDPB, methacryloyloxydodecylpyridinium bromide; 5-NMSA, $\mathrm{N}$-methacryloyl-5-aminosalicylic acid
\end{abstract}

\section{Discussion}

Adhesion is the procedure of forming an adhesive joint. The initial substrate is termed adherend and the material producing the interface is termed the adhesive. ${ }^{1}$ The functions of dental adhesives is to promote conservation of tooth structure and enable minimally invasive dentistry, reinforcement of weakened dentin or enamel, reduce marginal staining, reduce microleakag and may also reduce postoperative sensitivity when used appropriately. ${ }^{1}$ The adhesive system should be non-toxic, provide adequate bond strength, resist wear, water sorption, provide sufficient wetting capacity and color stability. ${ }^{2}$ The ultimate goal of a bonded restoration is to attain an intimate adaptation of the restorative material with the dental substrate. This task is difficult to achieve as the bonding process for enamel is different from that for dentin. That is, dentin is much humid and more organic content than enamel. This humid and organic nature of dentin makes bonding extremely difficult. ${ }^{3}$ The purpose of this article is to review the main concepts regarding various adhesive systems.

\section{Steps for good adhesion ${ }^{3,4}$}

1. Substrate surface should be cleaned. Once the surface is clean, its surface energy is high and it is more likely to adsorb material from environment such as moisture or saliva. Therefore surface must be protected and next step in bonding procedure should be proceeded.

2. Good wetting of adhesive to substrate by removal of smear layer to decrease the contact angle and increase their spread onto the surface.

3. Highly adaptation to substrate produces intimate approximation of the material to avoid air entrapment or other materials.

4. Intimate contact of adhesive to the substrate produces physical, chemical or mechanical bonding. For effective chemical bonding, distance between the adhesive and substrate must be less and a high density of new bonds must form along the interface. Because this is rarely seen, bonding of restorative materials involves mechanical bonding. Mechanical bonds (gross mechanical retention and micro mechanical retention) involve adhesive interlocking with surface irregularities.

5. The adhesive be well cured under optional conditions.

\section{Application of bonding system}

The bonding systems have a valuable application in dentistry such as: sealant, enamel and dentin bonding system, amalgam-bonding system, resin-composite cement, crown and bridge cement and orthodontic bonding system. ${ }^{5}$

\section{Principles of adhesion}

\section{Bonding of resins to tooth structure is a result of four potential mechanisms $s^{4,5}$}

a. Mechanical-penetration of resin and creation of resin tags within the tooth surface.

b. Diffusion-precipitation of substances onto the tooth surfaces to which resin monomers can bond mechanically or chemically.

c. Adsorption-chemical bonding to the inorganic component (hydroxyapatite) or organic components (mainly Type I collagen) of tooth structure.

d. A combination of the previous three mechanisms usually responsible for bonding with modern adhesives.

\section{Composition of dental adhesives}

Resin components: In order to assure a good covalent bond between the adhesive and resin composite, dental adhesives contain resin monomers that are similar to those in resin composite. They act as a backbone providing structural continuity and thus mechanical properties such as strength. Monomers are the key constituents of adhesives. Two kinds of monomers can be distinguished: crosslinkers (have two polymerizable groups) and functional monomers (commonly have only one polymerizable group). ${ }^{6,7}$ 
Initiator systems: Adhesive systems should be cured before the application of the composite, to obtain an optimal degree of conversion, thus good mechanical strength of the adhesive layer, and to prevent overly thinning of the adhesive resin layer by the application of the composite. ${ }^{6,7}$

Photo-initiators: Many compounds can dissociate into free radicals upon absorption of light energy, such as camphorquinone (CQ), 1-phenyl-1,2 propanedione (PPD). ${ }^{6,7}$

Chemical-initiators: The use of chemical initiators is usually restricted to cements and resin that cannot rely on light curing for polymerization. The most common initiator in self-curing resins is benzoyl peroxide (BPO) in conjunction with co-initiator tertiary amine. ${ }^{6,7}$

Inhibitors: Inhibitors added to dental resins are actually antioxidants that are able to scavenge free radicals originating from prematurely reacted initiators. The most frequently used inhibitors in adhesives are butylated hydroxytoluene. ${ }^{6,7}$

Solvent: The addition of solvents to resins is essential. The wet nature of dentine only allows good wetting when a hydrophilic bonding is applied. Their main function is to promote good penetration of the monomers in the collagen network of the demineralized dentin, thus improve the diffusion ability of resin. In adhesives, water, ethanol and acetone are the most commonly used solvents. ${ }^{6,7}$

Water: Water-containing adhesives difficult to remove from adhesive solutions as it take longer time to evaporate; it is capable of expanding the collapsed and shrunken collagen network. ${ }^{6,7}$

Ethanol: Its higher vapor pressure compared to water allows faster evaporation by air-drying thus adding to water. ${ }^{6,7}$

Acetone: Acetone-containing adhesives combine both hydrophobic and hydrophilic components. Its high vapor pressure, which is about four times as high as that of ethanol, is a main advantage. However, its high volatity may also lead to reduce shelf life of acetone-containing adhesives, by rapid evaporation of the solvent. These systems should be applied on demineralized wet dentin to avoid collagen collapse. ${ }^{6,7}$

Fillers: Fillers are added to modify the strength and viscosity of adhesives. Depending on their chemical composition, fillers can also provide in fluoride release and radio-opacity. Moreover, particle size affects penetration ability. ${ }^{6,7}$

Modifiers: Manufacturers sometimes add specific ingredients such as: glutaraldehyde, which have desensitizing effect results from denaturation of collagen in dentin and the occlusion of dentinal tubules, in addition to strong antibacterial activity. Methacryloyloxydodecylpyridinium bromide (MDPB) monomer with antibacterial effect, N-methacryloyl-5-aminosalicylic acid (5-NMSA) monomer has a salicyl group that is intended to chelate with calcium in order to obtain a desensitizing effect. Simple fluorine compounds could be also added. ${ }^{6,7}$

\section{Mechanics of dental adhesion}

Substrate: Adhesive systems must establish a bond to both the restoration and the dental hard tissue. Composite restoratives consist of a hydrophobic matrix in which different filler particles are embedded. Teeth are comprised of two very different substrates: enamel and dentin. Enamel is essentially $96 \%$ hydroxyapatite, crystalline calcium phosphate, and $4 \%$ organic material and water 3 whereas dentin consists of $70 \%$ hydroxyapatite, $20 \%$ collagen and
$10 \%$ water. Enamel is thus an essentially dry substrate, whilst dentin is moist. Adhesives therefore need to possess both hydrophobic and hydrophilic properties in order to establish a bond to both substrates. ${ }^{8}$

Smear layer: The smear layer refers to a layer of dental "debris" about 1 micron thick lying over the prepared sections of tooth after instrumentation. It may have a protective function as it lowers dentin permeability; however as it partly penetrates the dentin tubule it can pose a challenge to effective bonding. With early composite materials, it was observed that bonding agents that removed the smear layer achieved better retention rates in clinical trials than those that merely modified it. Removal of the smear layer appeared to be a prerequisite for adhesion to dentin, and remains a largely accepted concept. ${ }^{8}$

Etching enamel: Buonocore was the first to demonstrate the acid etch technique on enamel. It increases the surface area, by leaving an irregular white etch pattern. The enamel prisms of enamel are cut either transversely or vertically during preparation and a micro-retention pattern forms during etching because the central and peripheral parts of the prisms feature different degrees of acid-solubility. A resin-based fluid, aided by capillary action is then able to flow into the microporosities created. Monomers polymerize and become interlocked with the enamel as resin tags. A stronger acid or longer exposure to acid is required to obtain an optimal retentive pattern on enamel than is needed to expose dentinal collagen in dentin bonding. ${ }^{8}$

Etching dentin: Etching dentin enlarges the tubular openings, removes or dissolves the smear layer and demineralizes surface dentin. Demineralization of peri- and intertubular dentin results in a cup shaped expansion of the dentin tubules to a depth of approximately $10 \mu \mathrm{m}$, creating porous zones with exposed collagen fibrils. This is fundamental to achieving an effective bond. Initially etching dentin was problematic as the first adhesive materials were hydrophobic. They worked sufficiently on enamel, but were unable to penetrate and bond to dentin successfully. Modern hydrophilic resins however penetrate moist etched dentin surfaces and form a hybrid layer whereby resin tags extending into the tubule form a micromechanical bond. The hybrid layer seals the exposed dentin and is linked covalently to the composite restoration during polymerization of the first increment. ${ }^{8}$ Hybridized dentin is a mixture of adhesive polymers and dental hard tissues, differing from the original tooth structure at a molecular-level. The fundamental principle therefore of adhesion to tooth substrates is based on an exchange process by which inorganic tooth material is exchanged for synthetic resin. ${ }^{9}$

\section{Mechanisms of adhesion to enamel and dentin}

\section{The "Total-etch" or "etch-and-rinse" technique}

The "total-etch" term refers to the procedure whereby both enamel and dentin are etched before bonding. Total-etch adhesives involve an initial etching step with phosphoric acid which removes the smear layer and conditions the preparation. The total-etch technique is also often referred to synonymously as the "etch-and-rinse" technique. The phosphoric acid is rinsed off together with the smear layer and the exposed dental tissue is carefully dried. Enamel is usually etched for longer than dentin. The "how wet is wet?" discussion refers to the necessity of not over-drying the dentin after etching and rinsing. Dentin should remain moist and slightly glossy in appearance, such that the collagen fibrils do not collapse as this would make the surface less permeable to hydrophilic monomers in the adhesive and create a weak interface, potentially leading to a poor bond and postoperative 
sensitivity. For this reason, plus the multi-step nature of the technique, total-etch adhesives are often referred to as technique-sensitive. They are however very well established and highly clinically successful. ${ }^{10}$

Selective-etch technique: This refers to the conventional etching technique whereby only the enamel edges of a preparation are etched with phosphoric acid and then rinsed. The dentin is then conditioned using an acidic primer step or an all-in-one self-etching adhesive. The smear layer is modified but not removed as surfaces are not rinsed after the primer application. This method (now less common than the total-etch technique) can also be seen as etch-and rinse method for enamel only. ${ }^{11}$

Self-etch technique: Self-etch adhesives are intended for use without a separate etching step. Self-etch systems contain acidic monomers that prime/etch the enamel and dentin. In contrast to total-etch systems there is less danger of excessive demineralization of the dentin because self-etch systems only demineralize dentin as far as the primer penetrates. Thus, all demineralized areas are immediately filled with monomer. The potentially technique-sensitive step of drying the dentin to just the right degree after etching is also not required thus the danger of collagen-fiber collapse can be excluded. Each of these factors should reduce the risk of postoperative complaints. ${ }^{8}$ The self-etch approach can be further subdivided into a 'strong' $(\mathrm{pH}<1)$, an 'intermediately strong' ( $\mathrm{pH} \approx 1.5)$, a 'mild' $(\mathrm{pH} \approx 2)$, and an 'ultramild' ( $\mathrm{pH} \geq 2.5$ ) self-etch approach depending on the self-etching or demineralization intensity. ${ }^{12}$

\section{A Brief history of adhesives}

Michael Buonocore ${ }^{13}$ is widely considered to be the first person to propose the application of adhesion technology in dentistry. His groundbreaking research in 1955 demonstrated for the first time that acid-etching of enamel could provide a surface suitable for bonding with resins. By the mid-1960s, the first commercially available pitand-fissure sealants and composite resin materials utilizing this new adhesive technology were used clinically. Buonocore theorized that resin tags filling the defects created by the etchant were responsible for enamel adhesion, and by the late 1960s, he also proposed that bonding to dentin was possible. Since then, dental adhesives have been developed that provide numerically higher bond strengths and more substantive bonded interfaces to both enamel and dentin. By the 1980s, etch-and rinse adhesives had gained widespread acceptability. By the 1990s, the concept of the "hybrid layer" was accepted, and both multi-step and single-step adhesives were available. ${ }^{8}$

\section{Classification of adhesives}

Over the years adhesives have been classified variably according to generation, method of etching and the number of bottles involved or the number of individual steps necessary for the entire bonding procedure. In addition to this, dentists often define generations differently, they may or may not include etching in calculating the number of bottles or steps involved and some authors allocate specific adhesives to different groups e.g. the classification of a multi-step adhesive with a separate primer (traditionally viewed as an etch-andrinse adhesive) as a self-etch adhesive. ${ }^{5}$

\section{According to generation (chronological development)}

Dental adhesives can to a degree be categorized chronologically according to generation, a historical system of identification commonly used by adhesive manufacturers. The generation simply refers to when and in what order this type of adhesive was developed by the dental industry, ranging from 1st generation in the 1960s to modern 7 th generation adhesives. 1st and 2nd generation bonding agents are no longer used, due mainly to failed attempts to bond with a loosely bound smear layer. They achieved poor bond strengths of 2-8 MPa and failed to prevent marginal gaps. Manufacturers currently produce so-called 7 th generation products, however 3rd, 4th, 5th and 6 th generation adhesives remain popular and offer various advantages depending on the clinical situation and the clinician's personal preferences and experience. The approximate timescale and principle differences between generations are shown in Table $1 .^{5-16}$

Table I Classification of adhesives according to generation, mechanism of adhesion and number of clinical steps ${ }^{5-8}$

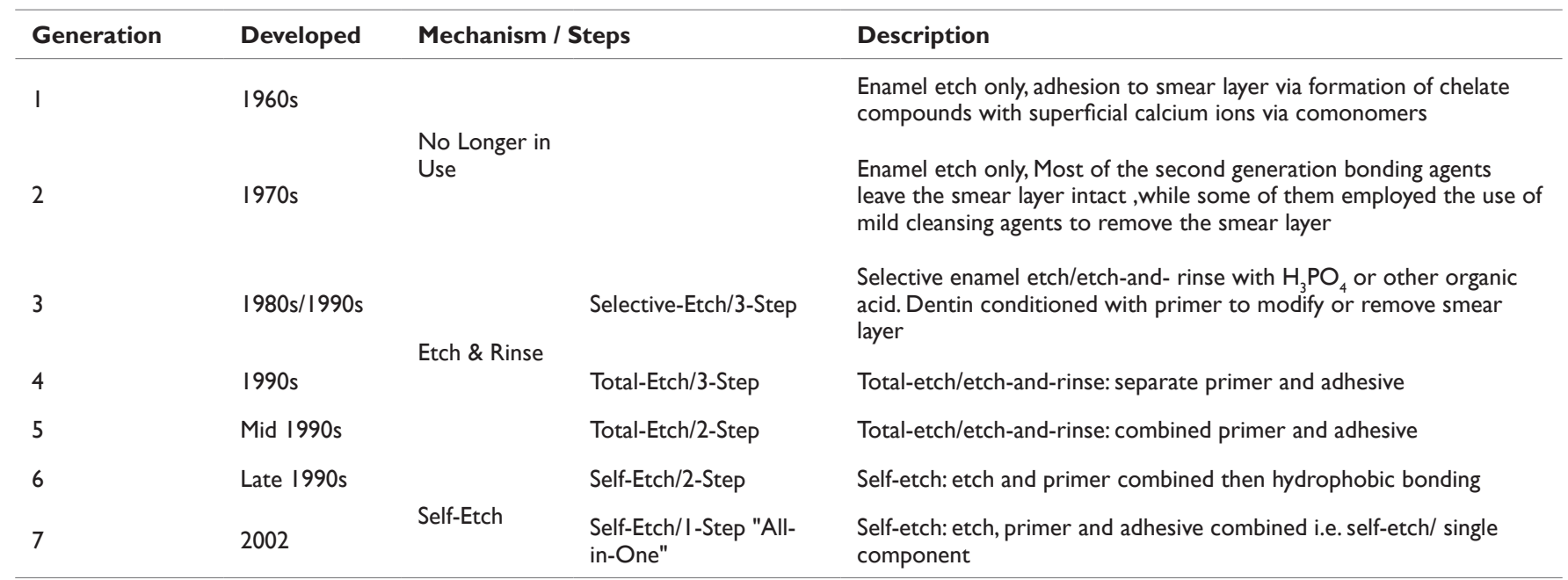

\section{According to clinical application steps}

Modern dental adhesives can be classified into two basic types: etch-and-rinse and self-etch adhesives. Although the etch-andrinse term is often used as alternative to total-etch adhesives. These systems can then be sub-classified based on the number of clinical steps involved: e.g. three-step and two-step etch-and-rinse systems and two-step and one-step self-etch systems..$^{5-16}$ The etch-and-rinse system has a separate etch-and-rinse step before the priming and bonding steps. The 3-step etch-and-rinse/total-etch system (using fourth generation adhesives) follows the conventional "etch-rinseprime-bond" approach. The two step etch-and-rinse system (using 
fifth-generation adhesives, also known as one-bottle adhesives) combines the primer and the bonding agent into one application. The self-etch adhesive system eliminates the rinsing phase after etching by using non-rinse acidic monomers to etch and prime dentin simultaneously. The two-step self-etch system (involving sixth generation adhesives) uses acidic monomers as self-etch primers in the initial step and an adhesive resin in the second step. ${ }^{16}$ The one-step self-etch system (using seventh generation adhesives, also known as all-in-one adhesives) combines the (self-etch) acidic primer with the adhesive resin in one application step. This allows for simultaneous infiltration of adhesive resin to the depth of demineralization, which may reduce postoperative sensitivity. ${ }^{6-16}$

\section{According to adhesion strategy}

Smear layer removing adhesive systems (etch and rinse approach or total etch approach)

These bonding agents completely remove the smear layer employing the total etch concept. They work on the principle of hybrid layer and resin tags. Enamel and dentin are etched simultaneously using an acid (preferably 37\% phosphoric acid). After washing and drying the tooth surface, primer and bonding agent are applied either separately or in combination. ${ }^{8}$

\section{Smear layer modifying adhesive systems}

In this strategy bonding agents modify the smear layer and incorporate it in the bonding process. According to these, the smear layer acts as a natural protective barrier to the pulp, protecting it against bacterial penetration and also limiting the outflow of dentinal fluid which can hamper the bonding process. Enamel is selectively etched with $37 \%$ phosphoric acid (taking care not to etch dentin). After washing and drying the tooth, primer and adhesive are applied separately or in combination. This results in micromechanical interaction of dentin and bonding system without exposure of collagen fibrils. $^{8}$

\section{Smear layer dissolving adhesive systems (self-etch approach)}

These agents partially demineralize the smear layer and the superficial dentin surface without removing the remnants of smear layer or the smear plugs. They make use of acidic primers also termed as self-etch primers or self-etch adhesives which provide simultaneous conditioning and priming of both enamel and dentin. After this, adhesive is applied without washing the tooth surface. The basis for use of these systems is to condition the dentin and to simultaneously penetrate to the depth of demineralized dentin with monomers which can be polymerized. ${ }^{8}$

\section{Glass-ionomer approach}

Glass-ionomers remain the only materials that are self-adhesive to tooth tissue. In general, polyalkenoic acid conditioner is applied for 10-20 seconds and rinsed off, followed by gently air drying without dehydrating the surface. The increase in bonding efficiency could be attributed to: ${ }^{17}$

1. Cleaning effect, by which loose cutting debris is removed.

2. Partial demineralization effect, by which the surface area is increased and micro-porosities for micro-mechanical interlocking or hybridization are exposed.
3. Chemical interaction of the polyalkenoic acid with residual hydroxyapatite.

\section{Newly introduced components of dental adhesive}

MDPB (e.g. Adper prompt $\mathbf{L}$ pop $\square$ ): MDPB is a unique monomer that was developed to provide resin-based materials with long-lasting antibacterial activity without releasing the antibacterial agent. After curing, MDPB is covalently bonded to the polymer network and acts as a contact inhibitor against the bacteria that comes in direct contact with the polymer (6).

Meth acrylamides (e.g . AdheSE): Meth acrylamides have an amide $(-\mathrm{CO}-\mathrm{NH}-$ or $-\mathrm{CO}-\mathrm{N}-$ ) group instead of an ester group $(-\mathrm{CO}-\mathrm{O}-\mathrm{R}-)$ as in conventional acrylates and methacrylates, which promotes the formation of hydrogen bonds between the carboxyl and amide groups of the monomer with the carboxyl groups of collagen. ${ }^{6}$

\section{Nanofilled bonding agent (e.g. Nano-Bond)}

Nano-composites and nano-adhesives are one of the greatest contributions of nano dentistry which contain nano-sized fillers, which characterized by stable nano-filler that will not settle out of dispersion. Nano-particles prevent agglomeration thus producing high dentin and enamel bond strength, high stress absorption, longer shelf life, durable marginal seal, produce a low film thickness, show better penetration in dentin than other generations. ${ }^{5-12}$

\section{PEM-F (e.g. Xeno IV)}

Is a monomer with a grafted fluoride functional group. The rationale for this monomer is the release of fluoride upon admixture with water, which will scavenge calcium in order to intensify the demineralization reaction.

\section{NMSA (e.g. clearfil liner bond)}

Has a salicylic group that is intended to chelate with calcium in order to obtain a desensitizing effect. ${ }^{6}$

\section{Funding details}

None.

\section{Acknowledgments}

None.

\section{Conflicts of interest}

The author declares that there is no conflict of interest.

\section{References}

1. Craig R, Powers J. Restorative dental materials. $13^{\text {th }}$ ed. 2012:327-329.

2. Perdigao J, Geraldeli S, Hodges J. Total-etch versus self-etch adhesive: effect on postoperative sensitivity. The Journal of the American Dental Association. 2013;134(12):1621-1629.

3. Buch A, Choksi D, Idnani B, et al. Current concepts in dental adhesion : a review. Journal of dental sciences. 2012;3(1):21-35.

4. Heymann H, Swift E, Ritter A. Sturdevant's art and science of operative dentistry. th $^{\text {th }}$ ed. 2002:238-258.

5. Gupta N, Kathuria N, Gulati M, et al. "Bonding": Foundation of Dentistry. Journal of innovative dentistry. 2011;1(3):60-66. 
6. Van Landuyt K, Snauwaert J, De Munck J, et al. Systematic review of the chemical composition of contemporary dental adhesives. Biomaterials. 2017;28(26):3757-3785.

7. Silva e Souza MH Jr, Carneiro KG, Lobato MF, et al. Adhesive systems: important aspects related to their composition and clinical use. Journal of applied oral science. 2010;18(3):207-214.

8. Nisha G, Amit G. Textbook of operative dentistry. $2^{\text {nd }}$ ed. 2013:275-295.

9. Van Meerbeek, Bart, Vargas M, et al. Adhesives and cements to promote preservation dentistry. Journal of operative dentistry. 2001;6:119-144.

10. Hashimoto M, Tay FR, Svizero NR, et al. The effects of common errors on sealing ability of total-etch adhesives. Journal of dental materials. 2006;22(6):560-568

11. David S. Self-Etching Adhesives in the world of DBS. Journal of Iinside dentistry. 2011;7(3):1-5.

12. Van Meerbeek B, Peumans M, Poitevin A, et al. Relationship between bond-strength tests and clinical outcomes. Journal of dental materials. 2010;26(2):e100-e121.
13. Buonocore MG, Matsui A, Gwinnett AJ, et al. Penetration of resin dental materials into enamel surfaces with reference to bonding. Arch oral biology journal. 1968;13(1):61-70.

14. Nair M, Paul J, Kumar S, et al. Comparative evaluation of the bonding efficacy of sixth and seventh generation bonding agents: An In-Vitro study. J Conserv Dent. 2014;17(1):27-30.

15. Joseph P, Yadav C, Satheesh K, et al. Comparative evaluation of the bonding efficacy of sixth, seventh and eighth generation bonding agents: an in vitro study. International research journal of pharmacy. 2013;4(9):143-146.

16. Chandra S, Chandra G. Textbook of operative dentistry with MCQs. $1^{\text {st }}$ ed. 2007:220-231.

17. Meerbeek B, Yoshida Y, Inoue S, et al. Glass-ionomer adhesion: The mechanisms at the interface. Journal of dentistry. 2006;34(8):615-617. 